\title{
(En)quête de la construction du paysage olfactif de Montauban : projet Montalbanez - Écologie olfactive
}

\section{(In) quest of the construction of the olfactory landscape of Montauban:} Montalbanez project - Olfactory ecology

\author{
Émilie Bonnard ${ }^{1}$ \\ ${ }^{1}$ chercheur associée LARA-SEPPIA, Université de Toulouse - Jean Jaurès (emilie.bonnard@univ-tlse2.fr)
}

\begin{abstract}
RÉSUMÉ. Mon approche du paysage, en tant que designer olfactif, interroge le rapport de l'humain avec son environnement : l'écologie. À partir du projet Montalbanez : paysage(s) olfactif(s) de Montauban, je m'intéresse à quelques notions autour de l'écologie. Dans ce propos, la notion globale et floue d' " écologie ", succède à une période que l'on peut qualifiée de " proto-écologie " où l'on s'est essentiellement intéressée à des questions sanitaires. L'olfaction, sens chimique et communicationnel, relève de l'écologie en tissant des liens invisibles entre les individus et l'environnement. L'écologie olfactive : une " bioculture ", une culture vivante du vivant permet de traduire le monde invisible en monde perceptible en lui donnant forme. Cette première forme olfactive dominante de Montauban semble être une " note verte ". Cette senteur produite par la végétation chlorophyllienne semble envahir le paysage olfactif montalbanais appelé aussi « ville-campagne ».

ABSTRACT. My approach to the landscape, as an olfactory designer, questions the relationship of humans with their environment: ecology. From the Montalbanez project: olfactory landscape(s) of Montauban, we focus on some notions around ecology. In our remarks, the global and fuzzy notion of "ecology" follows a period that can be described as "proto-ecology" where we have mainly focused on health issues. The chemical and communicative sense of smell is ecologist by weaving invisible links between people and the environment. Olfactory ecology: a "bioculture", a living culture of the living allows to translate the invisible world into a perceptible world by giving it shape. This first olfactory form of Montauban seems to be a dominant "green note". This scent produced by chlorophyllous vegetation seems to invade the Montalban olfactory landscape also called "city-countryside".

MOTS-CLÉS. parfum, senteur, écologie, paysage, olfactif, Montauban.

KEYWORDS. perfume, scent, ecology, landscape, olfactory, Montauban.
\end{abstract}

Le paysage: une représentation d'un pays, d'un territoire, d'un espace, est appréhendé essentiellement par la vue, sens privilégié de notre culture, mais on peut aussi le saisir par l'odorat. Les senteurs perçues pourront être utilisées pour réaliser de nouveaux projets. Mon approche interroge le rapport de l'humain avec son environnement, c'est-à-dire l'écologie. L'expression "paysage olfactif ${ }^{1}$ » semble peu usitée, pourtant on trouve quelques descriptions de paysages olfactifs dans la littérature : Le ventre de Paris (1873), d'E. Zola décrit les senteurs de la capitale au $\mathrm{XIX}^{\mathrm{e}}$ siècle, Marcel Proust et Jean Giono multiplient ce genre de descriptions. La parfumeur Sissel Tolaas déambule dans l'espace urbain $\left(\mathrm{XX}^{\mathrm{e}}-\mathrm{XXI}^{\mathrm{e}}\right.$ siècle) pour capter les effluves in-situ et les réinterpréter dans ses parfums. Mais lorsque l'on est artiste plasticienne et designer, et que l'on a suivi des études en arts visuels comment aborder «l'écologie olfactive »? Cette intervention s'intéressera à mon projet de design olfactif Montalbanez.

\footnotetext{
${ }^{1}$ Poiret Nathalie, "Variation sur les paysages olfactifs ", dans Ambiances architecturales et urbaines, Lola verde paysage http://lolaverde.fr/wp-content/uploads/2014/09/Ambiances_Web.pdf
} 
Après une brève présentation du projet, nous nous intéresserons aux principales notions autour de l'écologie. Elle s'enchaîneront du général au particulier: l'écologie, puis la proto-écologie, l'écologie chimique, l'écologie olfactive, et enfin la « note verte ».

\section{Présentation du projet Montalbanez}

Depuis septembre 2018 : je cherche les senteurs de Montauban pour élaborer un/des paysage(s) olfactif(s) de cette ville, hier et aujourd'hui, pour demain. Le point de départ de ce projet, une observation : un presque désert ou néant olfactif, suspect pour une spécialiste de l'olfactif, et en même temps, un parfum «neutre » socialement acceptable pour la population. Ce projet s'élabore à partir d'une toile/page blanche. La première étape du projet consiste à sentir, percevoir les senteurs, les nommer, les collecter. L'accumulation de signes informatifs, souvent considérés comme des détails insignifiants, invisibles, pour les autres, parfois intimes, relève du quotidien, de la banalité. Je reconstitue progressivement, par petites touches, un patrimoine olfactif souvent évaporé, mais riche, discret, incrusté dans les mémoires, les souvenirs. Progressivement je réalise une représentation vague, une peinture abstraite qui se teinte de quelques nuances, quelques touches colorées qui éveillent des impressions, puis l'accumulation de détails permet de distinguer des formes, construire un paysage. La représentation devient figurative lorsque l'on a assez d'indices pour reconnaître un jardin, une rue. L'ajout de détails supplémentaires permet alors de créer des scènes " réalistes », voire « hyperréalistes ${ }^{2} »$. Je peux m'intéresser à un panorama, ou au contraire, faire des effets de zoom, me focaliser sur un détail. Par exemple, aujourd'hui, au moulin de la Palisse, le parfum de la farine, activité de minoterie du XVIII ${ }^{\mathrm{e}}$ siècle, a été remplacé par la senteur du bois du plancher, de la terre des murs, de l'humidité apportée par la proximité de la rivière car l'inodore électricité exacerbe les senteurs des matériaux qui constituent le lieu. Ainsi, je crée progressivement ou je fais apparaître, un/des paysages(s) olfactif(s) en construisant des représentations qui interrogent notre rapport à l'environnement, à l'histoire, à la culture, au temps et à la mémoire, c'est-à-dire à notre imaginaire à la fois personnel, individuel, collectif, populaire, et scientifique. Ce travail de recherche proustien actualise le passé dans le présent. Le but de ce projet consiste à dessiner ce paysage, non pas grâce à des mots, des crayons de couleur ou de la peinture, mais grâce à des parfums, et remplacer l'expérience visuelle habituelle, ou auditive, en expérience olfactive.

\section{Les différentes notions de l'écologie}

- L’écologie : une notion vague, floue

En 1866, le biologiste et philosophe Ernst Haeckel aurait été le premier à utiliser le mot « écologie » qu'il définit comme « la science qui étudie les rapports entre les organismes et le milieu où ils vivent $»^{3}$. Ce concept s'inspire des théories de Darwin développées dans De l'origine des espèces, publié en $1859^{4}$. Haeckel diffuse et popularise la théorie de Darwin, en énonçant la «loi de

\footnotetext{
${ }^{2}$ Cf Hyperréalisme : maîtres américains \& européens, présenté par Karel J. Geirlandt et Jean-Pierre Van Tieghem, Bruxelles, Isy Brachot, 1973
}

Hyperréalisme USA 1965-1975, commissaires d'exposition Jean-Claude Lebensztejn et Patrick Javault, Paris, Hazan : les Musées de Strasbourg, 2003

${ }^{3}$ «Écologie », Encyclopédie Universalis en ligne: https://www.universalis.fr/encyclopedie/ecologie/

${ }^{4}$ Darwin Charles, De l'origine des espèces, traduit de l'anglais par Clémence Royer, Paris, Guillaumin et Cie / V. Masson et fils, 1870 (1re édition 1859) https://gallica.bnf.fr/ark:/12148/bpt6k6564680d/f28.image 
la récapitulation », selon laquelle le développement embryonnaire serait une répétition de l'histoire évolutive ${ }^{5}$. Cependant, il existait des pratiques « écologiques » bien avant l'apparition de ce mot, on peut les qualifier de «proto-écologie ».

\section{- La proto-écologie : l’aspect sanitaire}

À Montauban dès la création de la ville, au Moyen Âge (en 1144), on trouve des règlements qui visent à réduire les odeurs urbaines pour des raisons sanitaires, voir notamment les règlements concernant les bouchers. La population avait déjà conscience que les odeurs influençaient notre rapport à l'environnement. Alain Corbin explique que la suppression des miasmes dans les villes aux XVII ${ }^{\mathrm{e}}$ et $\mathrm{XVIII}^{\mathrm{e}}$ siècles serait liée à une élévation du niveau sociale, dans l'imaginaire collectif : plus il y aurait d'odeurs, plus le niveau social serait bas. N'ayant pas (encore) trouvé ce genre d'information à Montauban, il semblerait que cela était plutôt dû aux regroupement des mêmes activités exercées dans un même quartier, et à l'entassement des boues et déchets, à la périphérie de la ville qui produisaient des émanations. Il faut sans doute attendre Lavoisier, et son Traité de chimie en 1789, pour comprendre la dangerosité des émanations de la décomposition des déchets à proximité des habitations car rien ne se perd, rien ne se crée, tout se transforme. Cet imaginaire semble perdurer. En effet, j'ai instauré un dialogue avec le personnel des archives municipales, départementales, et des musées de Montauban. Ceci à mis au jour un fossé culturel/scientifique : leur imaginaire classe les odeurs comme des traces de pollution, des déchets. On retrouve cet amalgame dans un célèbre texte local, écrit par le médecin Edmond Limayrac, en $1882^{6}$. Il décrit les odeurs insalubres du ruisseau Lagarrigue, et demande aux autorités locales, pour la santé publique, de recouvrir ce cours d'eau. Aujourd'hui, en circulant sur la rue de la Mandoune, on passe sur ce ruisseau dissimulé sous la chaussée. La logique du médecin consiste à cacher pour ne pas voir, et donc ne pas sentir. L'imaginaire « populaire » en ce début de $\mathrm{XXI}^{\mathrm{e}}$ siècle, semble imprégné par cette description olfactive, datée, du paysage local.

- L’écologie chimique : une communication silencieuse non muette

Le développement des sciences et des connaissances a permis l'individualisation de la chimie au $\mathrm{XVIII}^{\mathrm{e}}$ siècle, et le déploiement de ses compétences au XIX ${ }^{\mathrm{e}}$ siècle, en parvenant notamment au perfectionnement de la parfumerie, suite aux recherches de Chevreul (1786-1889) sur la couleur, puis de Perkin qui crée la mauvéine : le premier colorant industriel synthétique (1856), puis la coumarine : la première molécule synthétique de parfum $(1868)^{7}$. Le parfum, une odeur perçue à travers le prisme d'une culture, peut aussi d'un point de vue chimique cette fois, être définie comme un ensemble de molécules se déplaçant dans l'air. Les molécules étant invisibles à l'œil nu, elles s'adressent à un autre sens : l'odorat, le sens chimique. Ce sens permet de percevoir un monde « invisible », silencieux mais pas muet. Françoise Gail et Martine Hossaert-Mc Key expliquent que dans l'écologie chimique

«Une large diversité de molécules, allant de composés très simples à des mélanges très complexes, est impliquée dans la perception de l'environnement, dans la communication

\footnotetext{
${ }^{5}$ "Écologie », Encyclopédie Universalis en ligne: https://www.universalis.fr/encyclopedie/ecologie/

${ }^{6}$ Limayrac Edmond, Les odeurs de Montauban : le ruisseau Lagarrigue considéré au point de vue de la santé publique, Montauban, Guillau, 1882 (p.13-18) https://gallica.bnf.fr/ark:/12148/bpt6k6581216g.textelmage

${ }^{7}$ Chastrette Maurice, " Histoire et origine des parfums », dossier Chimie et beauté, CNRS, en ligne: http://sagascience.cnrs.fr/doschim/decouv/parfums/his_ori_par.html
} 
entre individus et dans les mécanismes de défense mis en place dans les interactions antagonistes. Ce domaine scientifique récent (1959) transdisciplinaire réussit le tour de force de réconcilier écologie et chimie ${ }^{8}$. »

L'écologie chimique étudie les interactions par molécules et substances interposées qui sont responsables d'attractions, répulsions, émotions, mémoires, c'est-à-dire de la régulation des comportements sexuels, alimentaires, sanitaires, sociaux... entre organismes : plantes, algues, bactéries, invertébrés et vertébrés ${ }^{9}$. Autrement dit, l'écologie chimique s'intéresse à la biodiversité dans toute sa complexité chimique interactionnelle ou communicationnelle. Il s'agit d'un réseau de dialogues, invisible à l'œil nu, mais concret, semblable au réseau virtuel d'internet. Le parfum constitue une partie de ce réseau de substances chimiques. La communication en jeu dans l'écologie chimique n'est ni verbale (auditive), ni écrite (visuelle), mais on peut utiliser notre langage verbal pour l'appréhender et l'interpréter, en la décrivant, la comparant à d'autres choses, etc.

- L'écologie olfactive : une bioculture ${ }^{10}$

Ma thèse Design olfactif: essence d'une voie de communication logographique (soutenue en 2014) tente d'expliquer le fonctionnement du langage olfactif, chimique, en le transposant en langage logographique grâce à la notion de «mot-objet » de Bertrand Russell ${ }^{11}$, et d'une catégorie de sinogrammes : les idéogrammes ${ }^{12}$. Pour résumer très rapidement, notre culture associe un parfum à une ou plusieurs idée(s) : des idéogrammes. Une phrase ou suite de parfums associe plusieurs idées, ce qui permet de produire du sens, une intention, selon le mode de penser taoïste. La transposition de la communication chimique en communication logographique, comme l'interprétation_: la reformulation des idées pour créer une signification identique, tend vers la traduction: une molécule correspond à un idéogramme. La transposition, l'interprétation et la traduction constituent une partie de ce qu'on pourrait appeler une « écologie olfactive ».

Mais le projet Montalbanez n'en est pas encore là... puisqu'il se situe dans une phase d'état des lieux, de relevés topo-aéro-graphiques de senteurs, et de recueil de données olfactives littéraires, iconiques, botaniques, historiques, géographiques, sociologiques, ethnographiques... Cette phase s'effectue in situ, et in naso, mais aussi par des relevés dans des livres, des témoignages, et surtout dans le dialogue avec des habitants ou des personnes qui travaillent à Montauban. Là encore, le rôle de la communication semble primordial, mais elle diffère de la communication chimique, même si elles se «superposent» souvent. La communication permet d'informer : de mettre un discours en forme pour créer des représentations. Le dialogue entre deux ou plusieurs personnes permet, à partir de plusieurs points de vue, plusieurs perceptions et ressentis, de constituer, de tisser un consensus,

\footnotetext{
${ }^{8}$ Écologie chimique : le langage de la nature, dir. Hossaert-Mckey Martine et Bagnères-Urbany Anne-Geneviève, Paris, Cherche midi, 2012, Avant-propos p.9

${ }^{9}$ id. p.12

${ }^{10} \mathrm{Cf}$ Gélard Marie-Luce, Les sens en mots, entretiens avec J. Candau, A. Corbin, D. Howes, F. Laplantine, D. Le Breton, G. Vigarello, Paris, Pétra, 2017, p.13 titre « Une anthropologie des sens bioculturelle »

${ }^{11}$ Russell Bertrand, Signification et vérité, traduit de l'anglais par Philippe Devaux, Paris, Flammarion, 2013 (1re édition 1969), p.105-106
}

${ }^{12}$ Les idéogrammes utilisent un seul caractère pour représenter une idée, ce qui correspond a une bouffée de parfum. Pour complexifier une idée ou la préciser, on peut lui juxtaposer un autre caractère. Concernant la classification des caractères chinois, voir Viviane Alleton, Jean François Billeter, Léon Vandermeersch ... 
une représentation partageable «commune », pour un groupe de personnes donné, à un moment donné, dans un endroit donné (unité de temps, de lieu, de personnes).

- La «note verte »

Un consensus semble apparaître, celui de la «note verte ». En effet, Montauban, qualifiée de « ville-campagne », semble associer des senteurs de la ville, c'est-à-dire des activités produites par l'humain, et des senteurs végétales. Dans les rues piétonnes du centre-ville grâce aux boutiques, on peut sentir le parfum des crêpes, du caramel, du savon, des viennoiseries, du fromage, du café... et celui de la végétation urbaine, continuum de la campagne environnante. Les arbres et les herbes semblent constituer l'essentiel de la végétation montalbanaise. La prédominance visuelle de cette verdure influence probablement la perception olfactive d'une note verte. Dans la parfumerie, la notion de «note verte » a surgi, au milieu du $\mathrm{XX}^{\mathrm{e}}$ siècle, du dialogue entre le philosophe Étienne Souriau, et le parfumeur Edmond Roudnitska qui a passé sa vie à élever la création artisanale du parfum au rang d'œuvre d'art. «Note verte ${ }^{13} »$ : cette expression typique poético-onirique du lexique de la parfumerie croise le vocabulaire de la musique et de la peinture. La note désigne un élément qui s'agence à d'autres éléments pour créer une composition (qui se développe dans le temps). E. de Feydeau précise que la note verte évoque la nature, de la feuille à la tige jusqu'à l'herbe ou le foin fraîchement coupés. Les parfums verts sont vifs et fusants. La fraîcheur du vert est rendue grâce à la menthe, au thé vert, au galbanum, au pin, au sapin, à l'eucalyptus... La note verte a été créée pour reproduire, imiter la nature, souvent à partir de molécules synthétiques, c'est-à-dire fabriquées artificiellement par la chimie.

Mais revenons à Montauban, où l'on retrouve la menthe dans l'herbier d'Adrien Lagrèze-Fossat (XIX ${ }^{\mathrm{e}}$ siècle), conservé dans les archives du Muséum Victor Brun, mais elle se fait discrète dans la ville. Les pelouses et les arbres prennent plus d'ampleur. À Montauban, on trouve quelques pins et sapins, peu ou point de thé vert, de galbanum, d'eucalyptus. C'est pourquoi, il faudrait probablement recenser toutes les essences d'arbres pour obtenir la richesse des nuances de la note verte montalbanaise. On sait qu'à la création du cours Foucault, on planta uniquement des ormeaux (petits ormes), donc le parfum de ce jardin était uniforme, ce n'est plus le cas actuellement car l'expérience a montré que cela empêchait le bon développement des arbres. Aujourd'hui la politique de la ville prône la multiplication des essences : hêtres, peupliers, châtaigniers, érables... pour favoriser la communication, et ainsi, la santé de la végétation et l'expansion de son parfum.

Le projet ne fait que commencer, il permet cependant d'introduire quelques notions d'écologie qui seront à développer plus longuement en fonction de l'évolution du projet. Les notions comme le projet restent à affiner. Toutefois, on peut observer que l'écologie semble liée à une sensibilisation de et par l'expérience sensorielle donc corporelle, ainsi qu'à une mise en mots(-images) qui permettent de construire une représentation : un paysage olfactif.

\footnotetext{
${ }^{13}$ Feydeau Élisabeth (de), Les parfums : histoire, anthologie, dictionnaire, Paris, Robert Laffont, 2011, p.1111, Vent vert de Balmain (1945) est considéré comme l'archétype des parfums « verts ».
} 


\section{Bibliographie indicative :}

Corbin Alain, Le miasme et la jonquille, Paris, Flammarion, 20016 ( $1^{\mathrm{re}}$ édition 1984)

Écologie chimique : le langage de la nature, dir. Hossaert-Mckey Martine et Bagnères-Urbany Anne-Geneviève, Paris, Cherche midi, 2012

Gélard Marie-Luce, Les sens en mots, entretiens avec J. Candau, A. Corbin, D. Howes, F. Laplantine, D. Le Breton, G. Vigarello, Paris, Pétra, 2017

Géographie des odeurs, dir. Robert Dulau et Jean-Robert Pitte, Paris, L'Harmattan, 1998

Lao-Tseu, Tao-to king, traduit du chinois par Liou Kia-hway, Paris, Gallimard, 2010 (1 ${ }^{\mathrm{re}}$ édition 1967)

Le parfum de Nantes, dir. Aude Casseyre, Saint-Sébastien-sur-Loire, Orbestier, 2013

Limayrac Edmond, Les odeurs de Montauban : le ruisseau Lagarrigue considéré au point de vue de la santé publique, Montauban, Guillau, 1882

Mathis Charles-François et Pépy Émilie-Anne, La ville végétale, Ceyzérieu, Champ Vallon, 2017

Roger Alain, Court traité du paysage, Paris, Gallimard, 1997

Roudnitska Edmond, L'esthétique en question : introduction à une esthétique de l'odorat, Paris, PUF, 1977

Russell Bertrand, Signification et vérité, traduit de l'anglais par Philippe Devaux, Paris, Flammarion, 2013 (1 ${ }^{\mathrm{re}}$ édition 1969) 